\title{
CAMELoT - Tradutor Semiautomático de Processos em BPMN para Modelos Compreensíveis aos Cidadãos
}

\section{CAMELoT - Semiautomatic Translator of BPMN Processes for Models Understandable to Citizens}

\author{
Rodrigo Oliveira $^{1}$, Cláudia Cappelli $^{2}$, Flávia Santoro ${ }^{2}$ \\ ${ }^{1}$ Programa de Pós-Graduação em Informática (PPGI) da Universidade Federal do Rio de \\ Janeiro (UFRJ), Rio de Janeiro, RJ, Brasil \\ ${ }^{2}$ Instituto de Matemática e Estatística (IME) da Universidade do Estado do Rio de \\ Janeiro (UERJ), Rio de Janeiro, RJ, Brasil \\ rodrigo.oliveiralppgi.ufrj.br, claudia.cappelli@gmail.com, \\ flaviadime.uerj.br

\begin{abstract}
Business process models are artifacts used to support understanding of how to get a service or product, but as they are built with technical languages, they are not easy for citizens to understand. Therefore, a "citizen" process language needs to be used, however, it is unproductive for organizations to model all their processes again using this new language, as the use of BPMN is more common. Therefore, an automated translation is necessary, and in this article, we describe the CAMELoT application that translates business process models semi-automatically into models in a citizen process language, which seeks to facilitate citizens' understanding.
\end{abstract}

Keywords: Business Process Management; Transparency; Understandability; Citizen Language.

Resumo. Modelos de processos de negócios são artefatos usados para apoiar o entendimento de como obter um serviço ou produto, porém, como são construídos com linguagens técnicas, não são de fácil compreensão pelos cidadãos. Por isso, uma linguagem de processos "cidadã" precisa ser utilizada, contudo, é improdutivo para as organizações modelar todos os seus processos de novo usando essa nova linguagem, uma vez que é mais comum o uso de BPMN. Logo, uma tradução automatizada é necessária, e neste artigo, descrevemos o aplicativo CAMELoT que traduz de forma semiautomática modelos de processo de negócio para modelos numa linguagem de processos cidadã, que busca facilitar o entendimento dos cidadãos.

Palavras-Chave: Gerenciamento de Processos de Negócios; Transparência; Compressibilidade; Linguagem Cidadã.

\section{Introdução}

Transformações digitais estão gerando o estabelecimento de uma sociedade com cidadãos cada vez mais participativos, com capacidade de entender e acessar a informação disponível gerando uma elevada demanda por serviços transparentes

Cite as: Oliveira, R, Cappelli, C., Santoro, F. (2021) CAMELoT - Semiautomatic Translator of BPMN Processes for Models Understandable to Citizens (CAMELoT - Tradutor Semiautomático de Processos em BPMN para Modelos Compreensíveis aos Cidadãos). iSys: Revista Brasileira de Sistemas de Informação (Brazilian Journal of Information Systems), 14(3), 5-24. DOI: 10.5753/isys.2021.998 
(Holzner, 2006). Além disso, legislações regulamentam o direito ao acesso a informações produzidas ou detidas pelo Governo como a Lei de Acesso no contexto brasileiro (LEI No 12.527, DE 18 DE NOVEMBRO DE 2011), que estipula a garantia da transparência informacional das instituições públicas. Por meio disso, estabelece-se uma alta necessidade de sistemas que gerem eficientemente meios de transmitir com clareza e simplicidade todas as informações relevantes nestas instituições.

A modelagem de processos de negócios pode ser usada para atender a esse princípio de transparência na esfera pública e privada, pois são artefatos que podem ser usados para apoiar o entendimento de como obter um produto ou serviço, inclusive apresentando informações importantes, tais como, a sequência de tarefas a serem executadas (quem é responsável por cada tarefa; restrições que são aplicadas a determinadas situações; bem como as entradas e saídas) (Ferreira, et al., 2010). A representação de processos é frequentemente feita através da Business Process Modeling Notation (BPMN), que provou ser uma notação padrão para muitas organizações. No entanto, como Silva (Silva, et al., 2016) concluiu, BPMN não é de fácil compreensão por não-especialistas, isto é, fora o âmbito gerencial, não é inerente ao cidadão o conhecimento sobre notações técnicas e a interpretação de diagramas, impedindo a transparência, clareza e facilidade de interpretação desses procedimentos.

Algumas propostas de Linguagem Cidadã baseada nas ideias e pesquisas de Leite e Cappelli (2010), Engiel (2014) e Silva et. al. (2016) foram apresentadas e nesses estudos realizados foi demonstrado que esta nova linguagem tem potencial de ser mais fácil de entender do que BPMN. No entanto, seria improdutivo para as empresas modelar tudo novamente usando essa nova linguagem. Portanto, argumentamos que uma transformação automatizada é necessária. Neste artigo, descrevemos o aplicativo CAMELoT que apoia a tradução de modelos de processo em BPMN para modelos em uma linguagem cidadã de forma semiautomática, gerando um ambiente com melhor entendimento sobre os processos organizacionais. Estes processos traduzidos podem ser disponibilizados aos cidadãos em geral interessados na compreensão de serviços ofertados por organizações. Foram feitos testes para tradução de processos de uma Universidade Pública Brasileira. Este aplicativo está disponível para uso da comunidade científica e de organizações através de uma aplicação web.

O presente trabalho está estruturado em seções e, além desta introdução, será desenvolvido da seguinte forma: na seção 2 apresentamos uma revisão dos trabalhos relacionados ao tema proposto, na seção 3 apresentamos uma breve descrição da metodologia utilizada, na seção 4 conceituamos a linguagem cidadã em detalhes, prosseguindo para a seção 5 com o uso da ferramenta. Em seguida, oferecemos uma prova de conceito e uma validação com usuários para avaliação da proposta na seção 6 e uma breve discussão dos resultados na seção 7, finalizando com as contribuições da pesquisa e sugestões de possibilidades de aprofundamento posterior com a perspectiva de trabalhos futuros na seção 8. Este trabalho gerou as seguintes premiações e reconhecimento: Projeto Premiado como a Melhor Pesquisa em Informática na $15^{\circ}, 16^{\circ}$ e $17^{\circ}$ Jornada de Iniciação Científica da Universidade Federal do Estado do Rio de Janeiro - UNIRIO (2016 -2018) e indicado como Trabalho Destaque do Prêmio Nacional de Iniciação Científica do Conselho Nacional de Desenvolvimento Científico e Tecnológico - CNPq (2018). 


\section{Trabalhos Relacionados}

Vários estudos (Joneidi, et al., 2019), (Figl, 2017), (Hipp, et al., 2014), (Ottensooser, et al., 2012), (Schrepfer, 2010), (Rosemann, 2006), analisaram uma melhor compreensão dos modelos de processo de negócio, mas sem focar no público, em vez disso, melhorando a compreensão da própria linguagem formal ou conceitual. Por conseguinte, muitos outros trabalhos discutem os fatores que influenciam a compreensão do conteúdo sintático de modelos de processos, resumidos por: finalidade do modelo (Reijers, et al., 2011), domínio do problema (Lakhotia, et al., 1993), modelagem e notação (Sarshar, et al., 2005), (Hahn, et al., 1999), (Agarwal, et al., 1999), a apresentação visual (Moher, et al., 1993), (Reijers, et al., 2011), e complexidade de modelo de processo (Turetken, et al., 2016), (Canfora, et al., 2005). Por conseguinte, outras pesquisas desenvolvem diretrizes para melhorar a compreensão dos modelos com sistemas de avaliação dessas regras (Corradini et al., 2018) ou até ferramentas para tradução em linguagem natural de processos (Qian, et al., 2019), (Rodrigues, et. al., 2016), porém como argumentamos a seguir podem gerar ambiguidades.

No cenário analisado, observou-se uma lacuna de pesquisa, pois, apesar de os itens de conhecimento do processo serem de interesse efetivo do público-alvo, como sendo os cidadãos, em geral, os modelos são construídos como documentos técnicos internos. Os cidadãos podem não estar interessados em detalhes técnicos, mas sim em procedimentos de compreensão, o que faz cada atividade, seus objetivos, regras e informações necessárias. Então, como traduzir este tipo de modelo para uma 'linguagem cidadã'? A contribuição deste trabalho é inédita ao apresentar modelos com foco no cidadão de forma transparente e suas técnicas para melhor compreensibilidade a partir da especificação e construção de um novo modelo de processo, via software tradutor, com base no modelo de processo de negócios original.

\section{Metodologia}

A Design Science Research (DSR) foi a metodologia escolhida por se encarregar da ciência do projeto e ter como resultado a criação de um artefato, neste caso um de sistemas de informação (SI) para tradução de processos. Adotamos o método Design Science Research Methodology (DSRM) proposto por Peffers et al. (2007) por operacionalizar os princípios do paradigma da DSR com um etapas bem estabelecidas e de amplo uso na área. Sua execução no contexto deste artigo é descrita em ordem nos subitens a seguir:

A. Identificação e motivação de problemas: Com os trabalhos iniciais de Engiel et. al. (2014) e Silva et. al. (2016) formalizando as diretrizes da linguagem cidadã, percebeu-se que essa nova modelagem não tinha apoio computacional, e que sua exceção manual ou em ferramentas gráficas livres era custosa e suscetível a erros. Por esse motivo, se instaurou a ideia da pesquisa em como desenvolver um software que pudesse auxiliar nessa tradução de uma notação para outra.

B. Definição dos objetivos de uma solução: Um estudo sobre o estado da arte em modelagem de processos de negócios e entendimento de processos foi a etapa inicial, além da especificação da BPMN e formatos de exportação dos principais 
elementos e estudos dos formatos gráficos da nova notação. Portanto, definiu-se a solução como um sistema de tradução semi-automatizada, ou seja, intercalando passos de tradução automática com outros que dependem do modelador a partir de um arquivo do processo inicial em BPMN, oferecendo funcionalidades que permitam a tradução segundo à Linguagem Cidadã.

C. Design e desenvolvimento: Uma análise básica de requisitos, casos de usos e diagramas apoiaram o desenvolvimento e codificação da ferramenta capaz de traduzir efetivamente um modelo de processo em BPMN para a linguagem cidadã (Oliveira et al., 2017). Uma prototipagem inicial serviu para testar a tradução de vários processos e verificar que os requisitos definidos atendiam a proposta do projeto. Mais tarde, em parceria com a Empresa Júnior da UNIRIO, uma nova versão da ferramenta para web foi construída e adaptada, seguindo os mesmos requisitos definidos nas etapas iniciais do projeto e do protótipo. Desenvolver uma versão para uso online, pode oferecer mobilidade, flexibilidade e utilização imediata para qualquer interessado. Essa versão encontra-se disponível no repositório livre através do link: https://github.com/RodOlive/CAMELoT e pode ser acessada também no endereço: https://camelot-5bf1b.firebaseapp.com/\#!/ferramenta.

D. Demonstração: Uma prova de conceito foi realizada para verificar a viabilidade da aplicação em traduzir modelos de processos BPMN em modelos de linguagem cidadã. A prova de conceito $(\mathrm{PoC})$ é um método normalmente utilizado para demonstração, ou seja, para verificar que certos conceitos ou teorias têm o potencial para aplicação no mundo real.

E. Avaliação: Com entrevistas guiadas, apresentamos o sistema para usuários para efetuarem a tradução para Linguagem Cidadã em modelos de processos, a fim de apurar a aplicabilidade, facilidade de uso, aceitação e melhorias para a solução.

\section{A Linguagem Cidadã}

Um dos desafios para a transparência são os limites do conhecimento como afirma Tapscott (2003), os processos de negócio representados por uma técnica de modelagem precisam de conhecimento prévio sobre sua sintaxe e semântica (van der Aalst, 2013) e os cidadãos não têm conhecimento inerente de notações técnicas e interpretação de diagramas. Por outro lado, os processos de negócios representados por modelagem textual em linguagem natural podem gerar ambiguidade na interpretação e não têm nenhuma formalização definida que garanta a uniformidade e padronização (Hofstede, et al., 2003), logo, uma linguagem cidadã deve ser projetada dependendo, principalmente, do público ao qual se dedica a comunicar, fornecendo informações úteis e necessárias para os cidadãos (Fung, et al., 2007), dado que a sua meta não é a representação de informação (por exemplo, atividades, regras, documentos, etc.) que não toca o público envolvido. Portanto, para ser completo e expressivo, não é recomendado o uso de uma linguagem com sintaxe simples e semântica informal (por exemplo, a linguagem natural), e justamente a ausência de elementos que garantam esses recursos aumenta a eficácia de entendimento para o cidadão comum, que está 
mais interessado em informações diferentes de algumas daquelas necessárias na modelagem de um negócio.

Para notações técnicas, eficácia, eficiência e pragmatismo são o foco, uma vez que pretendem representar com exatidão (eficácia), o quanto possível (eficiência), a realidade (pragmatismo) dos processos de negócio da organização (Pinggera, et al., 2015). Para a linguagem cidadã, com base nos princípios de transparência, o foco é sobre a eficácia, com isso a linguagem cidadã difere das outras categorias no sentido de que: a) pode ser ambígua e não permite análise semântica como linguagens formais; b) ela não tem a responsabilidade de representar totalmente, formal e expressamente negócios, como linguagens conceituais; c) não é uma linguagem técnica para a implementação. Assim, Engiel et. al. (2014) e Silva et. al. (2016) adaptaram as operacionalizações de um catálogo de diretrizes, selecionando alguns métodos já utilizados para orientação de modelagem de processos mais compreensíveis e outros criados especificamente para essa linguagem cidadã, que são:

1.Não usar raias dentro dos modelos;

2.Usar a mesma cor para cada atividade de um ator;

3.Incluir grandes setas entre as atividades;

4. Retirar as atividades administrativas que não são de interesse do cidadão e não têm nenhuma influência no processo de entendimento;

5.Enumerar as atividades;

6.Escrever o texto mais explicativo e menos técnico;

7.Inserir caixas de comentário para informações essenciais relacionadas com atividades;

8.Não usar conectores lógicos.

9.Representar elementos que apresentem informações do processo, tais como bancos de dados ou documentos;

10. Remover eventos iniciais e eventos finais

A partir dessas operacionalizações, novos fluxos de modelos de processo foram construídos. A Figura 2 mostra o modelo de processo "Quebra de Requisitos de Disciplinas", traduzido para a Linguagem Cidadã, construído manualmente com base no modelo de processo apresentado na Figura 1. 


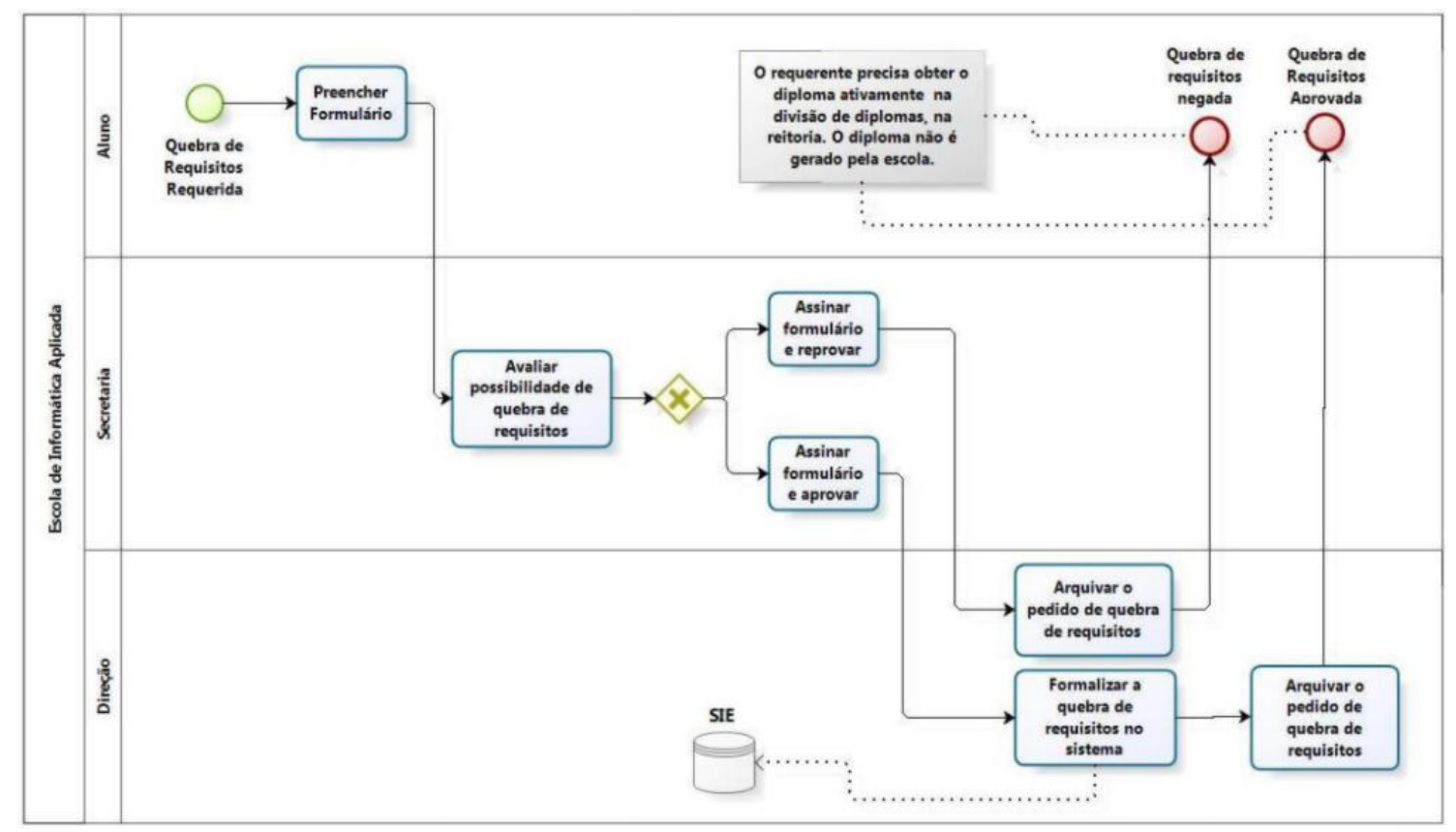

Figura 1. Processo de Quebra de Requisitos de Disciplina num curso de graduação de uma Universidade Pública brasileira Fonte: $\mathrm{O}$ autor.

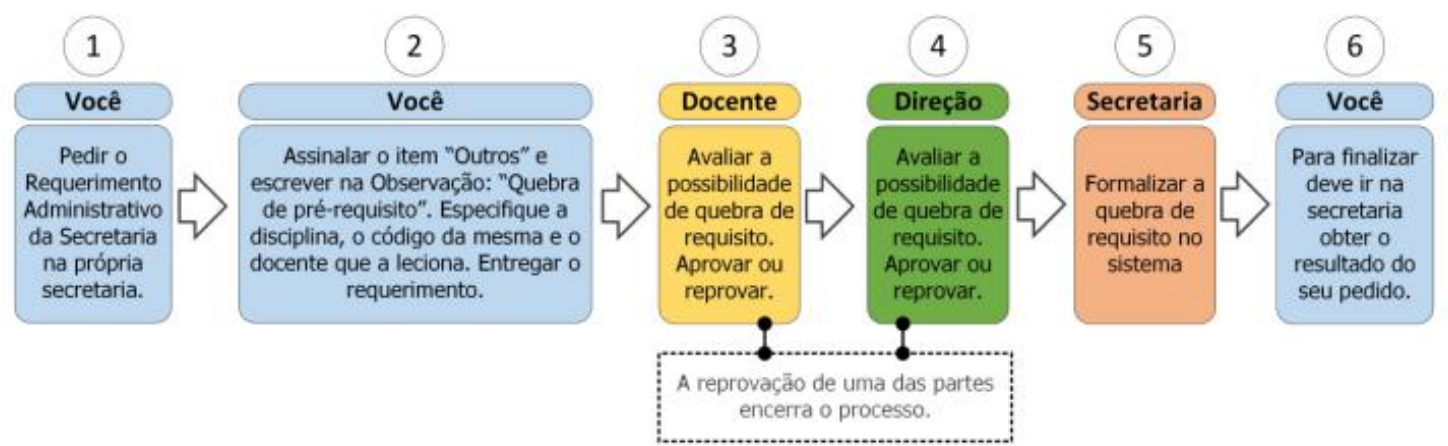

Figura 2. Processo de Quebra de Requisitos de Disciplina de um curso de graduação de uma Universidade Pública brasileira - representada em Linguagem Cidadã (Silva, et al., 2016).

Não foram apresentados procedimentos ou ferramentas de modelagem usando a Linguagem Cidadã, pois sabemos que as organizações costumam adotar padrões como BPMN, e que os modelos de processo não são destinados apenas para dar transparência aos cidadãos, mas sim, usados mais comumente para fins internos em uma organização. Este trabalho visa traduzir modelos de processos já construídos para um novo modelo, com o uso da Linguagem Cidadã, todavia, transformação manual implica em alto custo, difícil manutenção, além da não trivialidade de "tradução" de detalhes específicos, tais como, escolher o melhor conjunto de atividades relevantes para o cliente, simplificar a narrativa de atividades, escolher as regras mais relevantes para o processo, entre outros. Portanto, surge a necessidade de dispor de uma ferramenta que auxilie as organizações a traduzir seus processos já definidos em notações padrão para uma Linguagem Cidadã. 


\section{CAMELoT - Citizen Automatic Model Translator}

O sistema desenvolvido implementa os passos necessários para a tradução de um modelo de processo. Apresenta 4 etapas distintas:

1. Importação, etapa que recebe o processo a ser traduzido, verificando seu formato;

2. Análise, etapa que identifica os elementos do processo, e apresenta ao usuário uma visualização de uma modelo inicial traduzido;

3. Predefinição, etapa onde o usuário realiza as modificações que julgar necessárias seja para alterar as descrições textuais das atividades, excluir atores ou atividades que julgar irrelevante ou inserir regras e observação que auxiliem na melhoria do entendimento;

4. Exportação, etapa que permite salvar o modelo pronto nos formatos disponíveis.

A entrada de dados é realizada através da importação de um arquivo extraído da ferramenta que foi utilizada para a construção do modelo em linguagem técnica. $O$ formato de arquivo escolhido deve ser XPDL (XML Process Definition Language). Este foi escolhido para a implementação inicial da ferramenta pois é uma linguagem utilizada pela maioria das ferramentas de modelagem de processos. Esta linguagem é derivada do XML (eXtensible Markup Language) que descreve os dados envolvidos na criação do diagrama em BPMN. Nele são transcritos em linguagem de marcação (tags), os dados sobre as atividades, eventos, transições, gateways, etc. Além de suporte aos dados gráficos e de implementação.

O objetivo direto da criação dessa linguagem criado pelo WfMC (Workflow Management Coalition) é fornecer um meio viável de integração entre ferramentas de modelagem e comunicação entre sistemas que utilizam o BPMN. Ainda que existam sistemas que geram outras notações de exportação e importação de modelos, seja uma externa ou definição própria, gerar sistemas que utilizam o XPDL pode fornecer flexibilidade aos usuários na utilização, pois tais sistemas suportam a mesma linguagem. A partir desse arquivo como entrada, o sistema identifica todos os elementos necessários para a tradução do processo, segundo a estrutura de mapeamento descrita na Figura 3. 


\begin{tabular}{|c|c|}
\hline Objeto Gráfico em BPMN & Mapeamento em XPDL \\
\hline $\begin{array}{l}\text { Quebra de } \\
\text { Requisitos } \\
\text { Requerida } \\
\text { Evento Inicial }\end{array}$ & 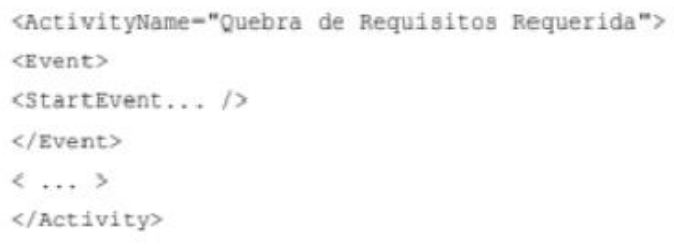 \\
\hline $\begin{array}{l}\begin{array}{c}\text { Preencher } \\
\text { Formulário }\end{array} \\
\text { Atividade }\end{array}$ & $\begin{array}{l}\langle\text { ActivityName-"Preencher Formulário"> } \\
\langle\ldots\rangle \\
\langle\text { Activity }\end{array}$ \\
\hline Evento Final &  \\
\hline 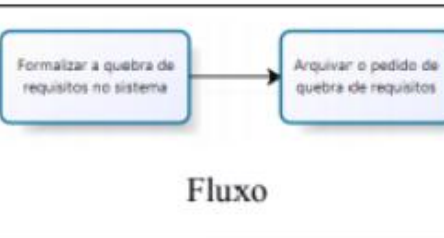 & $\begin{array}{l}\text { <Transition } \\
\text { Id-"f6565ac1-f026-4b95-930a-a305c8a904d9" } \\
\text { From-"e7547777-22c9-4eb7-886b-ddbeb219f41c" } \\
\text { To-"eda2a33e-a0c1-47f3-97a4-4f490875cd78"> } \\
\langle\ldots\rangle \\
\langle/ \text { Transition }\rangle\end{array}$ \\
\hline Gateway XOR & $\begin{array}{l}\langle\text { ActivityName-" "> } \\
\langle\text { Routeharkervisible="true" /> } \\
\langle\ldots\rangle \\
\text { </Activity> }\end{array}$ \\
\hline Gateway Paralelo & 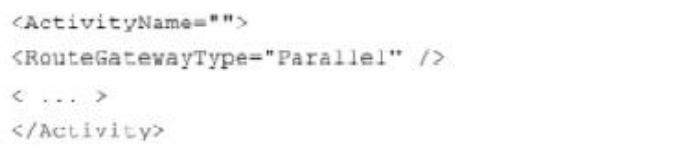 \\
\hline
\end{tabular}

Figura 3. Exemplos de Mapeamento de BPMN para XPDL. Fonte: o autor.

A tela inicial do sistema possui uma interface simples e intuitiva, com um design minimalista de símbolos e ícones para facilitar a compreensão. $\mathrm{O}$ usuário, ao clicar na seleção de arquivo, verá aberta uma janela de busca de arquivos com extensão em XPDL. Um filtro na busca não permitirá que outra extensão seja selecionada e um teste no arquivo verificará a versão compatível com o sistema, XPDL 2.2, conforme é destacado na Figura 4. O sistema irá identificar automaticamente os elementos fundamentais para a tradução: piscinas, raias (define os atores e as atividades associadas a eles), atividades, gateways (decisões e caminhos alternativos), fluxo (identifica a transição entre as atividades), comentários, entre outros, conforme a interface inicial do sistema. 


\section{CAMELOT \\ I Ferromento de traduçāo \\ (3) Sobre o projeto \\ 쯔 Contato}

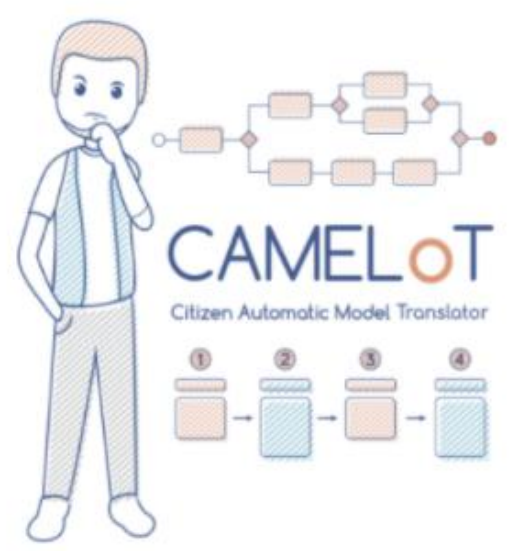

Vamos traduzir os seus modelos de processo de negócio para a linguagem cidadâ:

Selecionar um Arquivo

- Somente arquivos XPDL (Arquivo padrão de transferêncio de informaçóes de processos em BPMN)

Figura 4. CAMELoT - Interface apresentando as atividades e atores do modelo original para serem traduzidas.

A aplicação realiza a tradução de forma semiautomática, com passos que dependem do modelador e outros que se executam automaticamente. Após a seleção do arquivo, apresentam-se em tela as atividades do processo, como na Figura 5, onde o usuário poderá decidir quais não farão parte do novo diagrama, podendo remover atividades ou atores, editar o nome de cada tarefa para dispor de uma descrição de texto livre mais clara e objetiva, além de poder definir uma cor para os atores (por default todas as atividades assumem a cor branca). Contudo, é obrigatório pela sintaxe da linguagem que o usuário defina a cor padrão dos atores dos processos de sua organização ou uma paleta coerente com o seu público. Ao definir uma cor específica para um ator, todas as atividades assumem a cor do ator sobre sua responsabilidade, conforme a estrutura da linguagem cidadã apresentada anteriormente.

\section{CAMELOT

\section{Conteúdo do processo}

Todos os atores, tarefos e informoçs̃es do seu modelo estăo descritos no quadro abaixo, para modifico-los de acordo com a sua necessidade de entendimento para a Linguagem Cidada:


iSys: Revista Brasileira de Sistemas de Informação (iSys: Brazilian Journal of Information Systems) http://seer.unirio.br/index.php/isys/ 
Figura 5. CAMELoT - Interface apresentando as atividades e atores do modelo original para serem traduzidas.

Com todas as alterações já realizadas no passo seguinte, o sistema apresenta um diagrama do processo traduzido com todas as informações do modelo original mais as alterações realizadas pelo modelador no procedimento de tradução em uma prévisualização do novo modelo do processo, seguindo as especificações da linguagem cidadã mantendo a ordenação das atividades segundo o mesmo fluxo do processo original, com a numeração na parte superior de cada atividade. $\mathrm{O}$ modelador poderá verificar se o modelo atende aos critérios de compreensibilidade e realizar possíveis alterações para melhorá-lo, tais como, inserir comentários, regras de negócio, fluxos alternativos ou observações externas ao processo, cada uma interligada a atividade a qual se refere e com uma definição de cor e traçado diferentes, conforme a Figura 6.

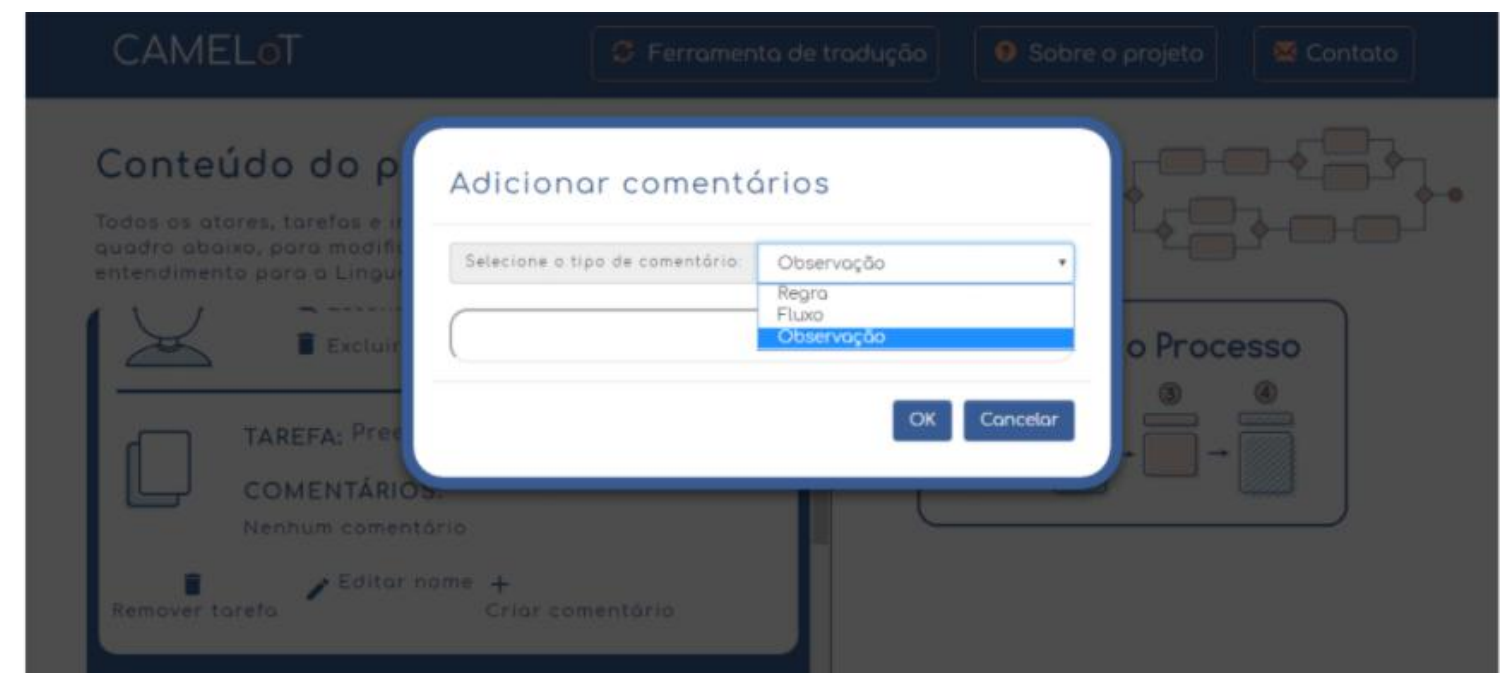

Figura 6. CAMELoT - Interface apresentando a inserção dos tipos de comentários em uma atividade específica.

A seguir apresentamos um exemplo de processo da secretaria da Escola de Informática de uma Universidade Pública brasileira que ilustra a transformação gerada pelo CAMELoT. A Figura 7 representa o processo modelado em BPMN e a Figura 8 a saída do sistema após a entrada com o arquivo XPDL e análises conforme descrito anteriormente. 


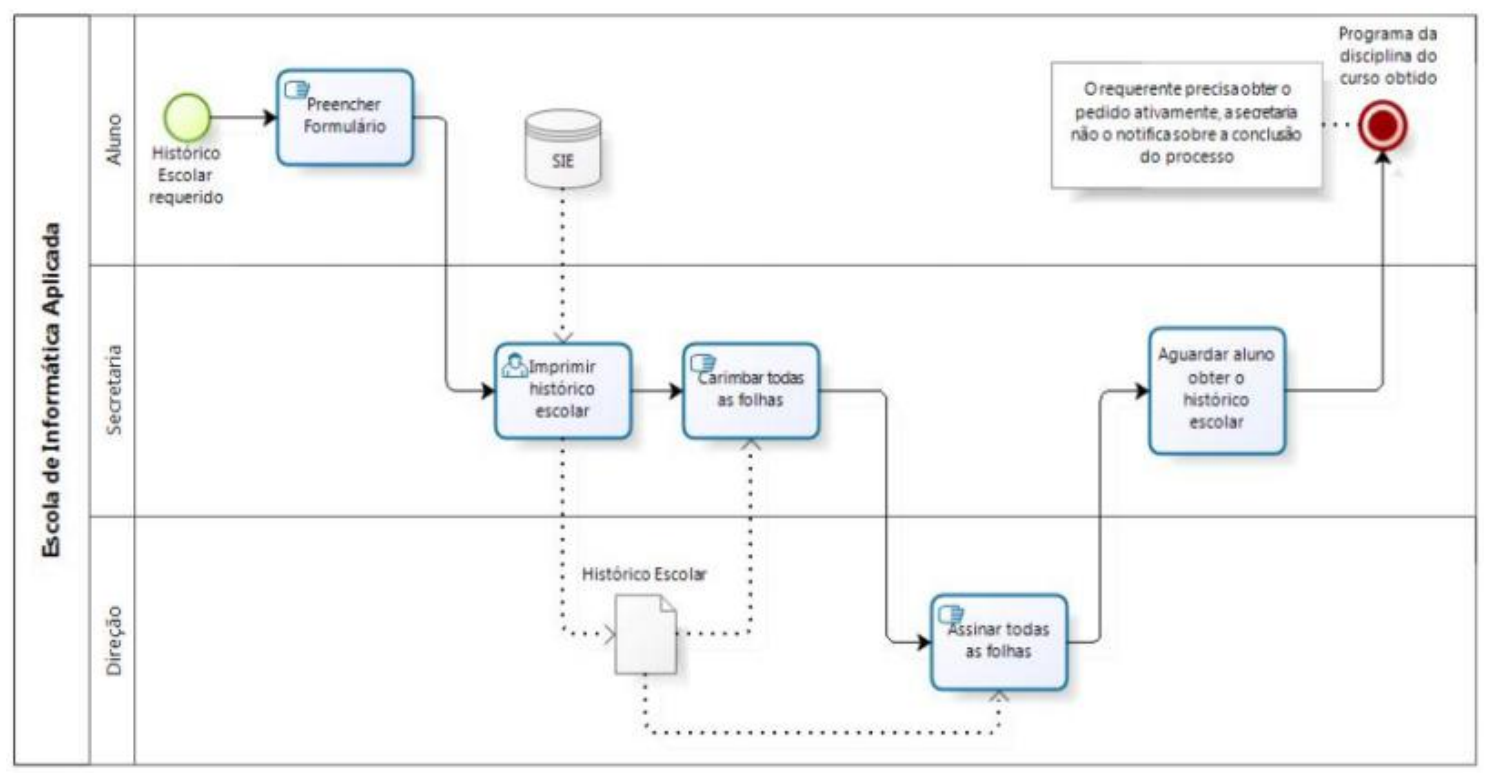

Figura 7. Processo Original em BPMN - Emissão de Histórico Escolar em uma Universidade Pública.
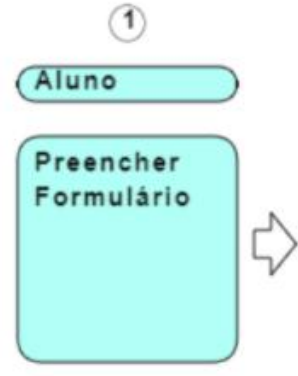

(2)



(3)

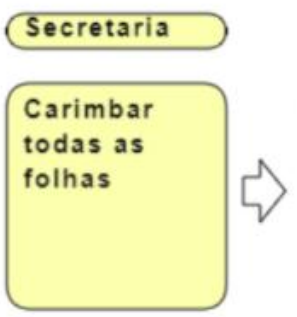

(4)

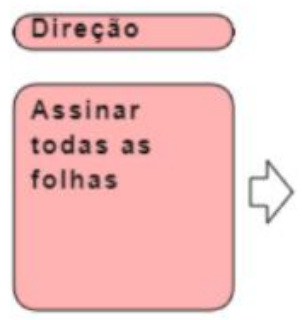

(5)

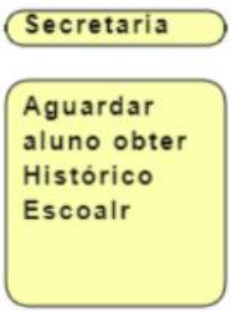

Figura 8. Processo traduzido via CAMELoT para Linguagem Cidadã

A ferramenta inclui todas as atividades em ordem e numeradas conforme o processo original. Eventos iniciais e finais são omitidos, assim como documentos e bases de dados. Neste exemplo, o modelador definiu cores para cada ator e com isso as atividades relacionadas a ele também ficam coloridas. É possível alterar as descrições de cada tarefa para oferecer mais clareza e expor mais detalhes de cada atividade ao leitor desse processo. Uma decisão interessante é pontuar quais dos atores é o alvo do entendimento do processo, um ator cliente, este seria o componente do processo a partir do qual é definida a visão de todas as atividades, ele será descrito como "você". Outra alteração possível é omitir ou unir tarefas para simplificar a visão do leitor, nesse caso as tarefas de carimbar e assinar todas as folhas poderiam ser únicas, mesmo que realizadas por atores diferentes, elas na visão do ator cliente, compreendem a mesma unidade de responsabilidade para emissão de histórico escolar. Unir estas tarefas trará maior simplificação do modelo e o tornará mais conciso. Também é possível deixar explícitas quais são as regras de negócio aplicáveis ao processo, ou seja, acrescentar detalhes em forma de comentários. Na Figura 9, apresentamos o modelo traduzido com as alterações finais que auxiliem o maior entendimento sobre como obter esse serviço. 


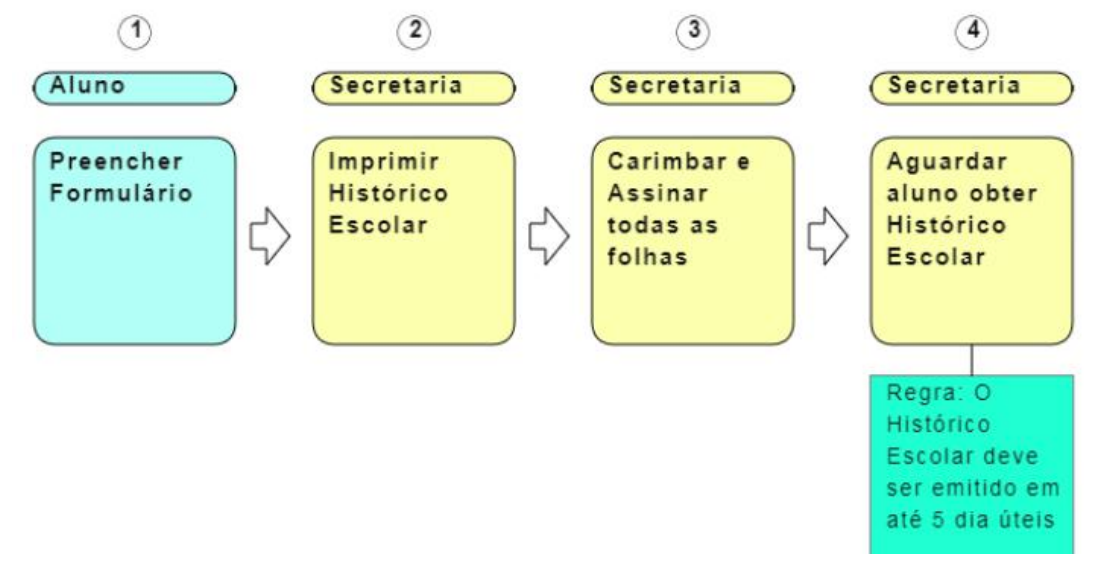

Figura 9. Processo traduzido via CAMELoT com alterações para maior entendimento.

\section{Demonstração e Avaliação}

Conforme previsto nas etapas do método DSRM (Peffers, 2007) um demonstração e avaliação deve ser executada, que neste caso consiste em uma prova de conceito e validação com usuários. Na primeira avaliação, foi testado se o procedimento de tradução é realizado corretamente pelas funcionalidades do sistema com processos reais. Enquanto a avaliação com usuários validou o software quanto à usabilidade e utilidade.

\subsection{Prova de Conceito}

O CAMELoT foi testado com 5 diferentes modelos de processo todos da secretaria escolar para verificar se o aplicativo era capaz de gerar novas visualizações dos modelos de processo, conforme definido nos requisitos linguísticos dos cidadãos, sem a introdução de erros e mantendo a ideia principal do procedimento descrito.

TABELA 1. Resumo dos resultados da PoC

\begin{tabular}{|c|c|c|c|c|c|c|c|}
\hline ID & Processo & $\begin{array}{c}\text { Atividades } \\
\text { Originais }\end{array}$ & $\begin{array}{l}\text { Atividades } \\
\text { Traduzidas }\end{array}$ & $\begin{array}{l}\text { Eventos } \\
\text { Originais }\end{array}$ & $\begin{array}{c}\text { Eventos } \\
\text { Traduzidos }\end{array}$ & $\begin{array}{l}\text { Gateways } \\
\text { Originais }\end{array}$ & $\begin{array}{c}\text { Gateways } \\
\text { Traduzidos }\end{array}$ \\
\hline 01 & Cancelamento de Matrícula & 4 & 3 & 2 & 0 & 0 & 0 \\
\hline 02 & Declaração de Matrícula & 4 & 3 & 2 & 0 & 0 & 0 \\
\hline 03 & Emissão de Histórico & 5 & 4 & 2 & 0 & 0 & 0 \\
\hline 04 & Quebra de Requisitos & 7 & 5 & 3 & 0 & 1 & 0 \\
\hline
\end{tabular}




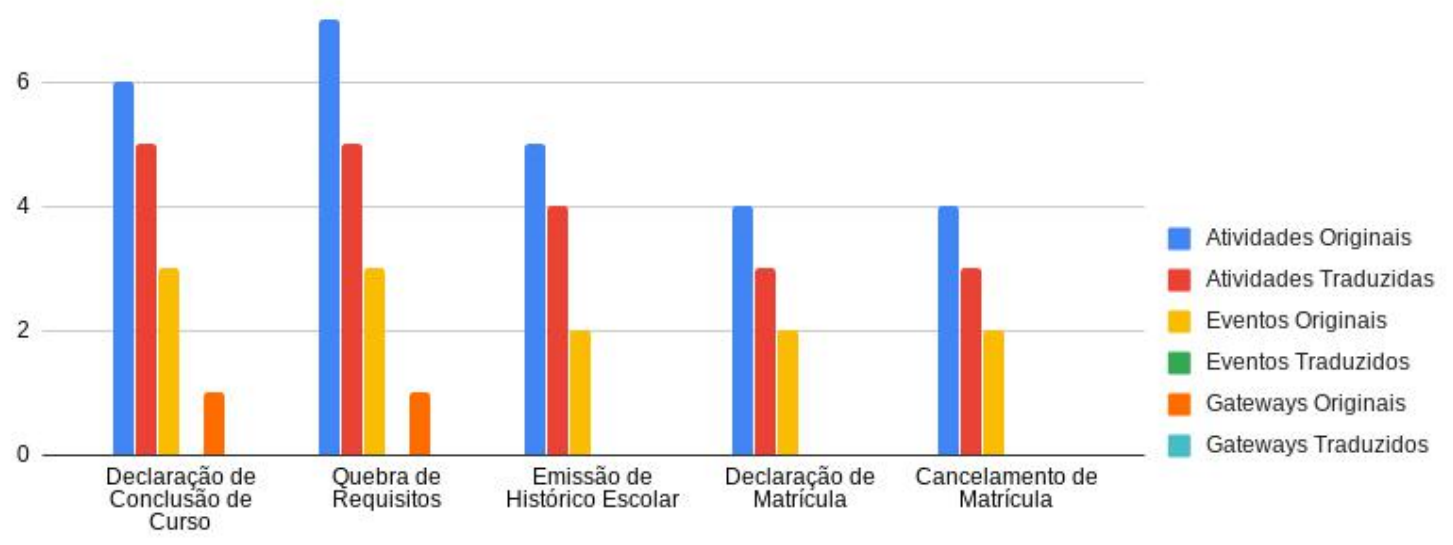

Figura 10. Gráfico - Resumo dos resultados da PoC

A Figura 10 apresenta a mesma informação em uma visualização gráfica que a tabela 1 mostrando que, em geral, o número de atividades é reduzido, e eventos e gateways desaparecem, diminuindo a complexidade do modelo de processo. Além desta prova inicial verificamos a tradução de outros processos da mesma secretaria que foram traduzidos de forma manual no primeiro momento (disponível em https://bsi.uniriotec.br/secretaria/), porém com algumas situações problema que serão discutidas nas limitações do trabalho. Contudo, vale ressaltar que a melhora no entendimento não vem somente a partir das reduções, mas como mostramos no exemplo de tradução na Figura 9, o sucesso na compreensão do modelo advém da habilidade do modelador em adaptar as atividades e incluir novos elementos para facilitar a compreensão por parte do leitor, algo que o sistema possibilita em sua funcionalidades, por isso se trata de um procedimento semiautomático conforme apresentado anteriormente, ou seja, há um custo adicional para o processo de tradução relacionado com as decisões manuais sobre quais atividades excluir e comentários textuais para incluir.

Argumentamos que essas linguagens destinadas a representar diferentes fins de modelos de processo não devem ser usadas para modelar processos a partir do zero, mas, em vez disso, elas devem ser geradas a partir de padrões (como BPMN) que já são utilizados por empresas. Daqui em diante, nós reivindicamos a relevância de uma aplicação para traduzir modelos. Esta noção é uma das principais contribuições desta pesquisa. Ao ler os modelos traduzidos na PoC, é possível comparar com o original. No entanto, embora não seja considerado grande o suficiente para ser um problema, há um custo adicional para o processo de tradução relacionado com as decisões manuais sobre quais atividades excluir e comentários textuais incluir. 


\subsection{Validação com Usuários}

Para validar a usabilidade e utilidade do sistema, foi realizada uma dinâmica baseada em entrevistas guiadas. O objetivo foi verificar a interação dos usuários com a ferramenta a fim de identificar problemas, novas funcionalidades que poderiam melhorar a experiência de tradução e definir os valores de aceitação do sistema. As entrevistas foram conduzidas de maneira virtual e semi-estruturada. A primeira parte dela consistiu em uma apresentação das diretrizes da Linguagem Cidadã conforme declaradas na seção 4, através de um exemplo de tradução em um processo genérico. Após isso na segunda etapa foi demonstrado como essa mesma tradução é executada pelo sistema em um processo real como exemplificado nas Figuras 7 e 8 . Todas as funcionalidades foram apresentadas seguindo os 4 passos de tradução já citados na seção 5. Na terceira etapa um processo real como na figura 1 foi entregue ao entrevistado no formato XPDL e uma imagem do modelo em BPMN para que o usuário pudesse simular uma tradução e operasse com as funções do sistema.

Por último, um formulário foi enviado ao entrevistado para que ele pudesse apresentar sua avaliação do sistema. O formulário foi construído a partir do modelo TAM - Modelo de Aceitação de Tecnologia (Davis, 1989). Nesse modelo, são medidos os graus de facilidade de uso, que é o quanto que o uso do sistema é realizado sem esforço, seguido pela utilidade percebida pelo usuário, ou seja, o quanto ele acredita que o uso do sistema melhoraria seu desempenho no trabalho. Por fim, a intenção de uso mede o quão aceitável é para o usuário indicar ou usar o sistema em ações futuras. Usando uma escala Likert de cinco pontos se perguntou pelo grau de concordância com as declarações listadas da tabela 3, de "discordo totalmente "(1) para" concordo totalmente "(5), com o "não concordo nem discordo" associado ao ponto (3).

TABELA 3. Declarações do formulário seguindo o Modelo de Aceitação de Tecnologia (TAM) (Davis, 1989). Fonte: Adaptado pelo autor.

Utilidade Percebida (UP)

UP1 Usar o CAMELoT me permite traduzir processos para linguagem cidadã mais rapidamente.

UP2 Usar o CAMELoT me permite traduzir processos para linguagem cidadã de forma mais fácil.

UP3 Usar o CAMELoT permite traduzir processos de forma correta segundo as regras da linguagem cidadã que foram apresentadas para você 
UP4 Usar o CAMELoT aumentaria minha produtividade se eu tivesse que traduzir muitos processos.
UP5 Usar o CAMELoT melhora meu desempenho se comparado a traduzir processos manualmente ou com
uma ferramenta gráfica.

UP6 Eu considero o CAMELoT útil para traduzir processos para linguagem cidadã.

Facilidade de Uso Percebida (FUP)
FUP1 É fácil aprender a usar o CAMELoT.
FUP2 Minha interação com o CAMELoT é clara e compreensível.
FUP3 Foi fácil se tornar habilidoso usando o CAMELoT.
FUP4 É fácil lembrar como realizar as tarefas no CAMELoT.
FUP5 Eu achei o CAMELoT fácil de usar!

Intenção de Uso (IU) IU1 Tendo a necessidade de traduzir um processo no futuro eu pretendo espontaneamente utilizar o
CAMELoT.

IU2 Trabalhando em organizações no futuro, eu recomendarei a utilização do CAMELoT para que eles traduzam seus processos.

Cada participante respondeu o formulário sem a intervenção do pesquisador, e somente após a aprovação de um termo de consentimento para com a pesquisa em curso. Além do preenchimento, foi solicitado que os entrevistados pudessem expor de forma audível o motivo de cada resposta para captar as motivações e questionamentos dos mesmos. No fim, dúvidas, sugestões de melhorias e impressões gerais também foram coletadas. Ao todo, foram 4 entrevistados, 3 homens e 1 mulher, dois deles na faixa de 20 a 30 anos e os outros entre 31 e 40 anos. Todos com nível superior na área da computação, dois deles pós-graduados também nessa área. Quanto ao nível de conhecimento acerca de processos de negócios e modelagem, dois deles atuam em projetos desta área em média por 3 anos e se declaram com um aprendizado avançado. Enquanto os outros dois nunca atuaram profissionalmente nesse campo, mas possuem conhecimento básico no assunto.

As respostas de cada entrevistado são apresentadas na Tabela 4, assim como a proporção das respostas de cada indicador medido pelo TAM, exibidos como uma pontuação em porcentagem.

TABELA 4. Resultados do formulário de cada entrevistado.

\begin{tabular}{|c|c|c|c|c|c|c|c|c|c|c|c|c|c|c|c|c|}
\hline & \multicolumn{6}{|c|}{$\begin{array}{l}\text { UTILIDADE } \\
\text { PERCEBIDA }\end{array}$} & \multicolumn{7}{|c|}{$\begin{array}{l}\text { FACILIDADE DE USO } \\
\text { PERCEBIDA }\end{array}$} & \multicolumn{3}{|c|}{$\begin{array}{l}\text { INTENÇÃO } \\
\text { DE USO }\end{array}$} \\
\hline & 1 & 2 & 3 & 4 & 5 & 6 & Total & 1 & 2 & 3 & 4 & 5 & Total & 1 & 2 & Total \\
\hline Usuário A & 5 & 5 & 4 & 5 & 5 & 5 & 29 & 5 & 5 & 5 & 5 & 5 & 25 & 5 & 5 & 10 \\
\hline
\end{tabular}




\begin{tabular}{|c|c|c|c|c|c|c|c|c|c|c|c|c|c|c|c|c|}
\hline Usuário B & 5 & 5 & 5 & 5 & 5 & 5 & 30 & 5 & 5 & 5 & 5 & 5 & 25 & 5 & 5 & 10 \\
\hline Usuário C & 5 & 5 & 5 & 5 & 5 & 5 & 30 & 5 & 5 & 5 & 5 & 5 & 25 & 5 & 5 & 10 \\
\hline Usuário D & 5 & 5 & 4 & 5 & 5 & 5 & 29 & 5 & 5 & 5 & 4 & 5 & 24 & 5 & 5 & 10 \\
\hline Total & & & & & & & 118 & & & & & & 100 & & & 40 \\
\hline Pontuação & & & & & & & $98 \%$ & & & & & & $99 \%$ & & & $100 \%$ \\
\hline
\end{tabular}

\section{Resultados e Discussão}

Todos os indicadores alcançaram pontuações altas, acima de 95\%, apoiando a aceitabilidade do sistema. Apenas uma das questões sobre utilidade obteve nota 4 de dois entrevistados, assim como uma questão sobre a facilidade de uso. A saber, a primeira se refere a precisão do sistema em traduzir processos na forma correta segundo as regras da linguagem cidadã que foram apresentadas no começo da entrevista. Os dois usuários se sentiram inseguros em caracterizar uma nota mais alta uma vez que não se aprofundaram nas regras da Linguagem Cidadã para atestar a corretude da tradução. Por se tratar de uma proposta de linguagem que ainda está se apresentando como modelo viável a transparência de processos, é natural não haver especialistas nessa nova abordagem. O único contato dos entrevistados com a linguagem foi através da apresentação desta no início da entrevista. Logo, é compreensível a colocação deste argumento pelos usuários.

A segunda pergunta que obteve nota inferior é a que se refere à facilidade de lembrar como realizar as tarefas no CAMELoT. A motivação desta pontuação também está associada ao conhecimento da Linguagem Cidadã. O entrevistado, por ter apenas um contato breve com as regras da linguagem, ao ser solicitada a tradução, se mostrou não recordar dos passos ou funcionalidades que deveria utilizar para realizar a tarefa. A maioria dos usuários se mostrou confiante na tradução, uma vez que poucos passos eram necessários e o sistema possui poucas funcionalidades para lembrança. Porém, um recurso que pode ser adicionado é apresentar na tela de análise, Figura 3, as principais regras da Linguagem Cidadã e as funcionalidades para alcançá-las, ou mesmo uma página a mais com um tutorial de fácil acesso ao usuário para relembrar as etapas da tradução.

As entrevistas foram altamente proveitosas para detectar alguns pequenos erros que permaneceram na fase de testes e também para percepção de funcionalidades que podem ser aperfeiçoadas ou a adição de novas para auxiliar ainda mais ao usuário. Por exemplo, um usuário percebeu que a cor escolhida de um ator desaparecia da interface ao trocar o seu nome. Alguns bloqueadores de anúncios ou extensões do navegador podem desabilitar funções do sistema, como excluir ou esconder tarefas. Foi percebido também que os campos de texto devem ser aumentados para evitar rolagem ao adicionar descrições das tarefas ou comentários que sejam grandes.

Quanto a funcionalidades é necessário adicionar um botão de exclusão de comentários, a possibilidade de reordenar as tarefas existentes e adicionar novas tarefas que não constam no modelo original. Para facilitar ao usuário, a inserção de uma 
visualização do modelo original em BPMN no próprio sistema ajudaria a comparar com a versão traduzida sem a necessidade de se retirar do sistema. Outra alteração importante é oferecer por default uma cor própria para cada ator do processo, atualmente nenhum deles é apresentado com cor definida logo após a inserção, permitindo que o usuário caia no erro de esquecer de definir uma cor. Portanto, uma vez apresentada uma cor padrão o usuário pode trocá-la ou permanecer com as inseridas automaticamente. Por fim, foi levantada a necessidade de exportar e/ou salvar o modelo em Linguagem Cidadã produzido em um formato que possa ser alterado futuramente. Se o processo sofrer alguma alteração, na versão atual da ferramenta,apenas uma imagem é salva, forçando ao usuário modelar novamente todo o processo, dificultando a reutilização.

\section{Conclusões}

Este artigo apresentou uma abordagem para gerar uma visualização amiga do cidadão de modelos de processos. A principal motivação para esta pesquisa é apoiar as organizações públicas para tornar seus processos disponíveis e compreensíveis para pessoas comuns. A premissa é que, embora as notações padrão sejam muito importantes, elas não são fáceis de serem aprendidas por não-técnicos. A linguagem cidadã foi proposta por Silva et al. (2016) para resolver este problema. Neste projeto argumentouse que o modelo gerado deve ser produzido automaticamente a partir de um padrão como BPMN, portanto, a ferramenta CAMELoT foi apresentada para transformação semiautomática de modelos de processos.

As primeiras impressões sobre a aplicação desta abordagem foram obtidas com a execução de uma PoC com modelos de processos em um contexto real e entrevistas com usuários. A ferramenta desenvolvida possui potencial para tornar a gestão de transparência das informações de empresas e instituições mais facilitada e atender prontamente a demanda de soluções práticas para aplicar transparência aos negócios. Porém, limitações do projeto foram identificadas num teste com processos mais elaborados, sobretudo quando há gateways aninhados, o aplicativo não foi capaz de gerar uma boa solução. Esse tipo de processo (com muitos pontos de decisão com base em regras sobre regras) seria provavelmente melhor representado com abordagens declarativas. Outro problema é sobre a dimensão no tamanho do modelo produzido pela simplificação que seria mais difícil de ser visualizada de forma horizontal (apenas uma dimensão).

Para trabalhos futuros é necessário implementar as correções e novas funcionalidades advindas das entrevistas com usuários, incluindo uma amostra maior dos respondentes. Testar o sistema em parceria com uma organização real com processos mais elaborados e buscar a composição da linguagem cidadã com textos estruturados para tratar esses processos de alto nível de complexidade. Por exemplo, a utilização das palavras "e" e "ou", diretamente no formato visual simplificado, para manter a supressão das imagens dos operadores sem perda de entendimento da sequência. Considerando também que a necessidade de inclusão de texto não aumentará demasiadamente a estrutura do processo simplificado. Outra questão é oferecer maior clareza em fluxos alternativos aninhados e lidar com o uso de eventos intermediários como tempo e mensagem, agregando a linguagem cidadã métodos declarativos para 
descrição em linguagem natural de forma a complementar o modelo ou integrando técnicas de animação (Gomes; Araujo, 2012).

\section{Referências}

Holzner B., Holzner L., Transparency in Global Change: The Vanguard of the Open Society. University of Pittsburgh Press; 1 edition, 2006.

Ferreira, J., Araujo, R., Baião, F .: Identificando Rupturas em Business-It Comunicação Através de Modelos de Negócios, na 12a Conferência Internacional sobre Sistemas de Informação Empresariais, Funchal, Madeira, Portugal, pp. 311325 (2010).

Silva, LP, Santoro, FM, Cappelli, C. Usando uma linguagem cidadão em modelos de processo público: o estudo de caso de uma universidade brasileira, em EGOVIS'16 (aceite para publicação) (2016).

Leite, JC, Cappelli, C .: Software Transparência, Negócios e Informação Engenharia de Sistemas, vol. 2, no. 3, Springer pp. 127-139 (2010).

Engiel, P., Araujo, R., Cappelli, C .: Designing Serviço Público Modelos de processo para a compreensibilidade, em Revista Eletrônica de e-Government, vol. 12, Issue 1, pp. 95-111 (2014).

Joneidi, J. M., SETAYESHI, S.: The Effect of Cognitive Style on the Understandability of Business Process Models. pp.111-134 (2019).

Figl, K. Comprehension of procedural visual business process models. Business \& Information Systems Engineering, pp. 41-67 (2017).

Hipp, M., Strauss, A., Michelberger, B., Bela, B., Reichert, M.: Enabling a UserFriendly Visualization of Business Process Models, in Business Process Management Workshops 2014, 3rd Int'l Workshop on Theory and Applications of Process Visualization (TaProViz'14). Eindhoven, The Netherlands (2014).

Ottensooser, A., Fekete, A., Reijers, H. A., Mendling, J.: Making sense of business process descriptions: An experimental comparison of graphical and textual notations, in Journal of Systems and Software, 85 (3), pp. 596-606 (2012).

Schrepfer, M.: Modeling Guidelines for Business Process Models. Master Thesis, Faculty of Economics and Business Administration, Humboldt State University (2010).

Rosemann, M.: Potential pitfalls of process modeling: part a, in Business Process Management Journal, vol. 12, no. 2, pp. 249-254 (2006).

Reijers, H.A., Mendling, J.: A study into the factors that influence the understandability of business process models, in IEEE Transactions on Systems Man and Cybernetics, Part A 41 (3), pp. 449-462 (2011).

Lakhotia, A.: Understanding someone elses code: Analysis of experiences, in Journal of Systems and Software, 23(3), pp. 269-275 (1993). 
Sarshar, K., Loos, P.: Comparing the control-flow of epc and petri net from the enduser perspective, in Business Process Management, 3rd International Conference, BPM 2005, Nancy, France. Proceedings, LNCS 3649, 2005, pp. 434-439 (2005).

Hahn, J., Kim, J.: Why are some diagrams easier to work with? Effects of diagrammatic representation on the cognitive integration process of systems 40 analysis and design, in ACM Transactions on Computer Human Interaction 6(3), pp. 181-213 (1999).

Agarwal, P. R., Sinha, A., Comprehending object and process models: An 761 empirical study, in IEEE Transactions on Software Engineering 25 (4), pp. 541- 556 (1999).

Moher, T., Mak, D., Blumenthal, B., Leventhal, L.: Comparing the Comprehensibility of Textual and Graphical Programs: The Case of Petri Nets, in Empirical Studies of Programmers: Fifth Workshop, pp. 137-161. Papers Presented at the Fifth Workshop on Empirical Studies of Programmers, Palo Alto, California. Norwood, USA: Ablex Publishing Corporation (1993).

Reijers, H.A., Mendling, J.: A study into the factors that influence the understandability of business process models, in IEEE Transactions on Systems Man and Cybernetics, Part A 41 (3), pp. 449-462 (2011).

Turetken, O., Rompen, T., Vanderfeesten, I., Dikici, A., \& van Moll, J.: The effect of modularity representation and presentation medium on the understandability of business process models in BPMN. In International Conference on Business Process Management, pp. 289-307 (2016).

Canfora, G., Garcia, F., Piattini, M., Ruiz, F., Visaggio, C.: A family of experiments to validate metrics for software process models, in Journal of Systems and Software 77(2), pp. 113-129 (2005).

Corradini, F., Ferrari, A., Fornari, F., Gnesi, S., Polini, A., Re, B., \& Spagnolo, G. O.: A guidelines framework for understandable BPMN models. Data \& Knowledge Engineering, 113, pp. 129-154 (2018).

Qian, C., Wen, L., Kumar A.: BePT: A Behavior-based Process Translator for Interpreting and Understanding Process Models. In Proceedings of the 28th ACM International Conference on Information and Knowledge Management (2019)

Rodrigues, R.A., Azevedo, L., Revoredo, K.: BPM2Text: Uma estrutura independente de linguagem para Modelos de Processo de Negócios para Texto em Linguagem Natural. iSys-Revista Brasileira de Sistemas de Informação, pp. 38-56 (2016).

Peffers, K., Tuunanen, T., Rothenberger, M. A., \& Chatterjee, S. (2007). A design science research methodology for information systems research. Journal of management information systems, 24(3), 45-77. 
Oliveira, R. D. S., Cappelli, C., \& Santoro, F. M. (2017). Transformação Semiautomática De Processos Baseados Em Bpmn Para Modelos Compreensíveis Aos Cidadãos. XVI. Jornada De Iniciação Científica, Unirio.

Tapscott, D., Ticoll, D.: The Naked Corporation: How the Age of Transparency Will Revolutionize Business. New York, USA: Free Press (2003).

van der Aalst, W.: Business Process Management: A Comprehensive Survey. Hindawi Publishing Corporation (2013).

Hofstede, A., van der Aalst, W., Weske, M.: Business Process Management: A Survey, in volume 2678 of the series Lecture Notes in Computer Science, pp 1-12. Berlin, Germany: Springer-Verlag (2003).

Fung, A., Graham, M., Well, D.: Full Disclosure: The Perils and Promise of Transparency. New York, USA: Cambridge University Press (2007).

Pinggera J., Soffer P., Fahland D., Weidlich M., Zugal S., Weber, B., Reijers H.A., Mendling J.: Styles in business process modeling: an exploration and a model, in Software and Systems Modeling, Vol. 14, No. 3, pp. 1055-1080. Springer Berlin Heidelberg (2015).

Davis, F. D. (1989). Perceived usefulness, perceived ease of use, and user acceptance of information technology. MIS quarterly, 319-340.

Gomes, J. M., \& Araujo, R. Promovendo a compreensão de regras em processos de prestação de serviços públicos utilizando a animação. in Anais do IV Workshop de Computação Aplicada em Governo Eletrônico (pp. 25-32). SBC. (2012) 